\title{
The Synchronization Design and Implementation of LTE-Advanced Real-Time Test Platform Based on Software Defined Radio
}

\author{
Peng $\mathrm{Li}^{1,2}$, Yun-jian Jia ${ }^{1}{ }^{1}$, Fei Chen ${ }^{1}$, Pei-hua $\mathrm{He}^{1}$ and Hui-fang Fan ${ }^{1}$ \\ ${ }^{1}$ College of Communication Engineering, Chongqing University, Chongqing, \\ China \\ ${ }^{2}$ School of Electronic and Electrical Engineering, Chongqing University of Arts \\ and Sciences, Chongqing, China \\ unjian@cqu.edu.cn
}

\begin{abstract}
To meet the needs of future mobile communication, an essential approach is adopting new architectures of the mobile communication network and advanced technologies of wireless transmission. We choose GNU Radio and Universal Software Radio Peripheral (USRP) to construct a test platform for mobile communication technologies based on SDR. With the platform, a real communication conforming to LTE-Advanced physical layer specification is implemented by the PSS/SSS of LTE radio frame. The platform provides a tool to design, test and verify mobile communication technologies in real environment.
\end{abstract}

Keywords: LTE-Advanced, Test platform, Software defined radio, Synchronization

With the rapid growth of mobile communication terminals, mobile data traffic also shows a steeply increasing trend. Cisco forecast, up to 2018, the number of global mobile terminals will be 1.4 times of the world's population; Global mobile data traffic will be 11 times in 2013, and the compound annual growth rate is $61 \%$ [1]. In addition to the rapid growth of terminals and data traffic, there will be a dramatic expansion in the scenarios, application field, and business types of mobile communication. In the future, mobile communication will have characteristics like large capacity, high speed, density and mobility as well as low delay. To achieve the mobile communication with above characteristics, mobile communication technology should make further improvements basing on the fourth generation mobile communication $(4 \mathrm{G})$, so that it can meet the demand of the fifth generation mobile communication (5G).

To meet the need of future mobile communications, it is a dominant approach to adopt advanced wireless technology and new networks architecture. Modeling analysis, simulation and the test of platform are main ways to design, test and verify the advanced mobile communication technologies. In terms of simulation, the Tsinghua University, Beijing University of Posts and Telecommunications, and Southeast University respectively constructed a system-level simulation platform for the LTE via using C++ and MATLAB [2-3]. The Vienna University of Technology constructed the link-level and system-level simulation platform conforming to LTE-Advanced specification [4-5]. The construction of the simulation platform provides an approach for the research on LTE and LTE-Advanced technologies.

However, adopting merely simulation cannot meet the needs of designing and testing for future mobile communication technologies. Compared with simulation, the test platform with a combination of hardware and software has advantages of real channel environment and interference, rapid operating speed and high real-time. Therefore, it is necessary to develop a real-time test platform combining hardware and software so as to meet the needs of future mobile communication technologies. 
Considering the above facts, we choose GNU Radio and Universal Software Radio Peripheral (USRP) to construct a test platform for mobile communication technologies based on SDR. In the process of building the test platform, we analysis and summarize the requirements of testing for the technologies of advanced wireless transmission as well as the architectures and technologies of new mobile communication network. To meet the testing requirements, we design the structure and working mode of the platform, realizing the functions of involved components and modules, besides, programming the GUI. Operating results show that the test platform achieves the synchronization of the receiver and the transmitter. The testing results faithfully reflect the influence on the synchronization of the platform by channel environment and interference.

\section{GNU Radio}

GNU Radio is an open source software. Operated on general-purpose processor, GNU Radio realizes the baseband signal process, including modulation, demodulation and operations relative to waveform of signal [6-7]. In GNU Radio, an object-oriented high level programming language, Python, can be used to program the signal processing flow chart, which is composed of several signal processing modules with specific functions.

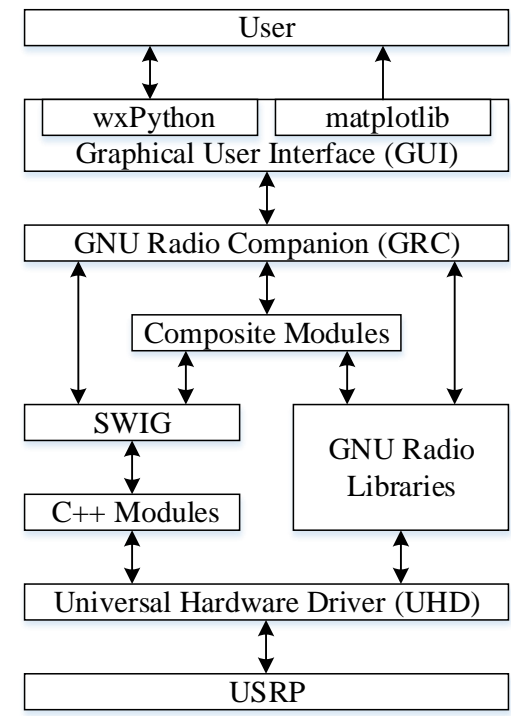

Figure 1. The Structure Diagram of GNU Radio Programming

As shown in Figure 1, Python is used to program the top-level module of the GNU Radio. The top-level module calls the underlying module via Simplified Wrapper and Interface Generator (SWIG) $[8,9]$. In the platform, $\mathrm{C}++$ is adopted to program signal processing module, which has strict requirement for real-time. And the graphical signal processing flow chart is programmed by GNU Radio Companion (GRC), similar to Simulink, a graphical interface tool.

\section{USRP}

USRP is composed of a FPGA mother board and one or several daughter board covering different frequency range so as to complete general-purpose operations, such as digital up/down convert, sampling and interpolation. USRP supports multiple software platforms, for example, GNU Radio, Labview and Matlab/Simulink. Compared with other Software Radio Peripherals like HackRF and bladeRF, USRP has advantages of broad bandwidth, high sampling precision, rapid sampling rate and supporting full duplex [10, 11]. In addition to a long transmission distance of USRP, its transmission rate reaches up 
to $1 \mathrm{Gbps}$ by adopting ethernet interface. Therefore, USRP can be used as radio frequency front-end for the test platform.

\section{The Procedure of Signal Processing at the Transmitter and Receiver}

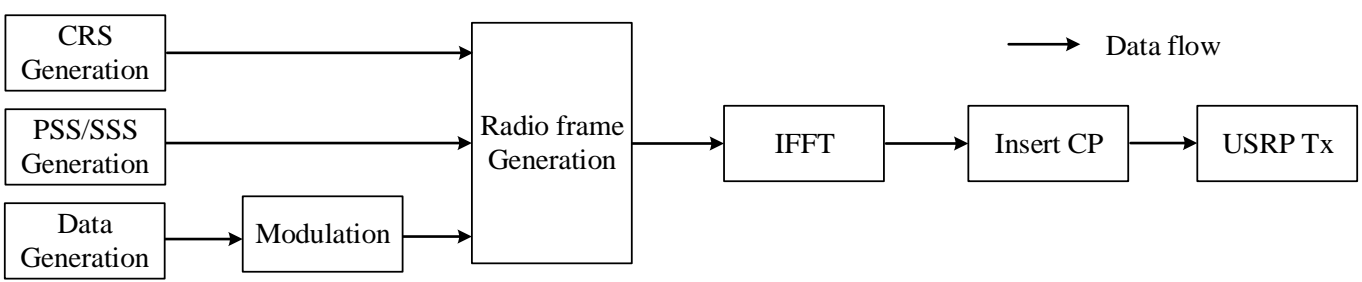

Figure 2. The Procedure of Signal Processing at the Transmitter

Figure 2 shows the signal processing flow of the transmitter of the test platform. Firstly, data and signals are generated, including Cell-specific Reference Signal (CRS), Primary Synchronization Signal (PSS) and Secondary Synchronization Signal (SSS). Secondly, Quadrature Phase Shift Keying (QPSK) modulation is used to modulate the data. Thirdly, LTE radio frame is generated according to the specification of resource mapping. Fourthly, the transmitter makes Inverse Fast Fourier Transform (IFFT) on radio frame, inserts Cyclic Prefix (CP) into the front of the data, generating Orthogonal Frequency Division Multiplexing (OFDM) symbol. Finally, the generated radio frames are transmitted one after another as OFDM symbol by USRP Tx.

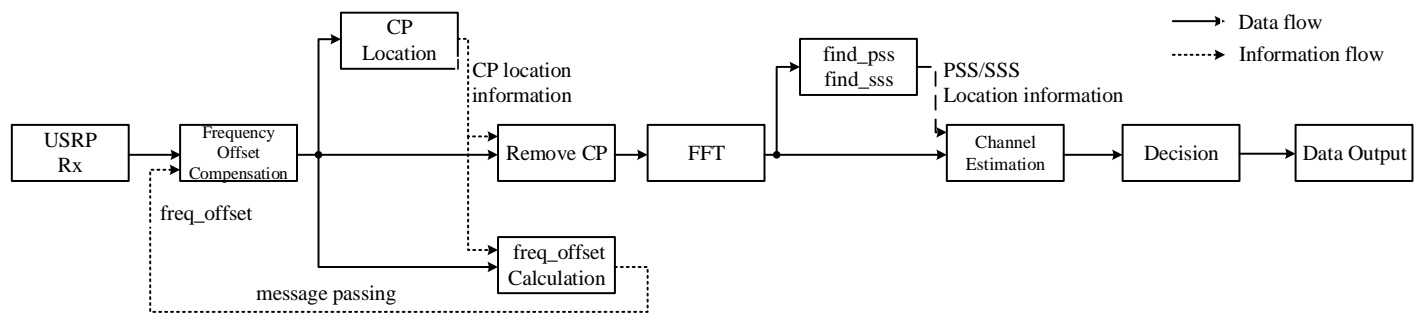

Figure 3. The Procedure of Signal Processing at the Receiver

Figure 3 shows the signal processing flow of the receiver of the test platform. The solid line represents the data flow while the dotted line is the information flow. After receiving radio frames, the USRP Rx corrects the frequency offset calculated by module at first. Through message_passing, the frequency offset is fed back to the module for correcting it to achieve the correction of the received data. Then the $\mathrm{CP}$ is located to get its location information, which are used for removing $\mathrm{CP}$ and calculating the frequency offset. After $\mathrm{CP}$ being removed, the data are transformed from time-domain to frequency-domain by Fast Fourier Transformation (FFT). Next, the synchronization of transmitter and receiver can be achieved by utilizing PSS and SSS. Channel estimation is realized by Cell-specific Reference Signal (CRS), and the estimated results can be used to compensate the amplitude and phase of the received data. After that, the module decides the received data, and then outputs the decision in a certain data format. 


\section{The Synchronization of the Platform}

The synchronization of transmitter and receiver can be achieved by using LTE physical synchronous signal. LTE physical layer is used for cell search, and then the user terminal can achieve synchronization and identification. The synchronous signals of LTE physical layer include PSS and SSS. Each synchronous signal lasts 1 OFDM symbol in time-domain, and occupies $1.08 \mathrm{MHz}$ of the central downlink bandwidth in frequency domain [12-14].

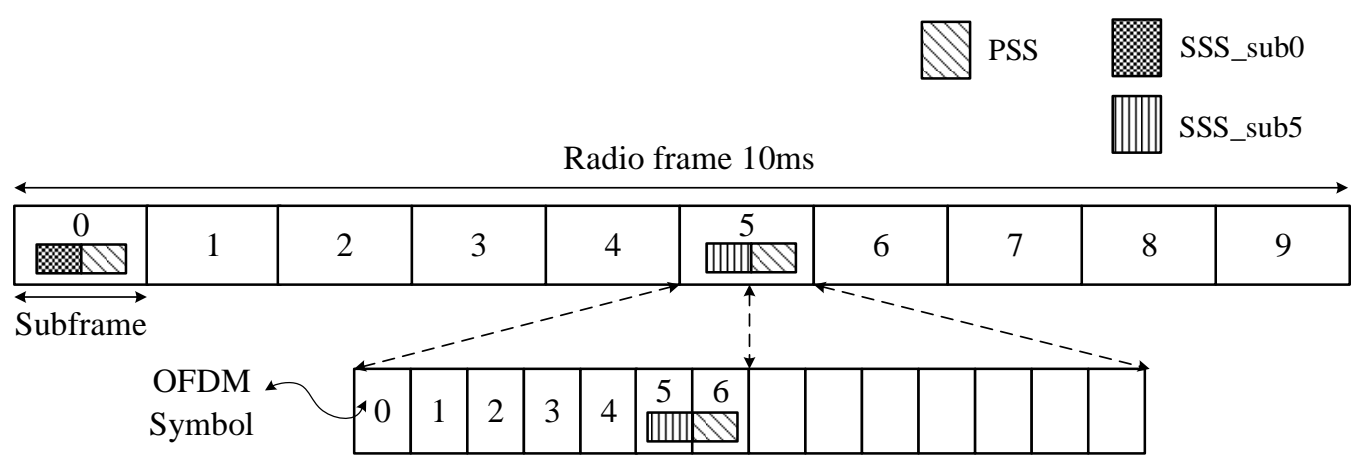

Figure 4. The Frame Structure of LTE FDD Type1

As shown in Figure 4, one radio frame comprises 10 subframes, numbered from 0 to 9. And one frame is composed of 20 slots, numbered from 0 to 19 , while each slot consists of 7 OFDM symbols, 0-6. Each frame comprises 140 OFDM symbols. It also has two PSS and SSS. And in one frame, two PSS are the same while the SSS are not for the first half frame, SSS_sub0, is different from the second half frame, SSS_sub5. SSS_sub0 is the SSS of number 0 subframe, while SSS_sub5 is that of number 5.

\subsection{Half-Frame Synchronization}

As shown in Figure 5, at first, PSS is used to achieve the half frame synchronization of transmitter and receiver. Then the receiver correlates the received 70 OFDM symbols one by one with the PSS generated in local receiver, getting 70 correlation values. PSS is included in the OFDM symbol corresponded to the highest correlation value, which should be numbered 6 consequently in the half-frame. However, the received 70 OFDM symbols are not exact half-frame. Therefore, we need Equation (1) to calculate the numbers.

Real number for OFDM symbol $=76$ - The number for OFDM symbol of current half-frame

After the procession of find_pss module, the receiver locates PSS. And beginning with the next half-frame, the receiver outputs the real number for OFDM symbol, achieving half-frame synchronization.

\subsection{Symbol Synchronization}

Then the symbol synchronization of transmitter and receiver can be achieved by using SSS. The symbol synchronization means that the receiver correctly numbers 140 OFDM symbols of each radio frame from 1 to 140 . The receiver uses the processing results of find_pss module, correlates the received OFDM symbol numbered 5 with SSS_sub0 and SSS_sub5 generated in local receiver respectively, and then stores the correlation values into Corr_f_0 and Corr_f_5. If Corr_f_0 > Corr_f_5, the half frame including current OFDM is the first half, then the number generated from the find_pss module is directly output. Otherwise, it is the second half, and then the generated number should be shifted by half frame. After the consecutive procession of the find_pss and find_sss modules, the 
symbol synchronization of transmitter and receiver is achieved at last.

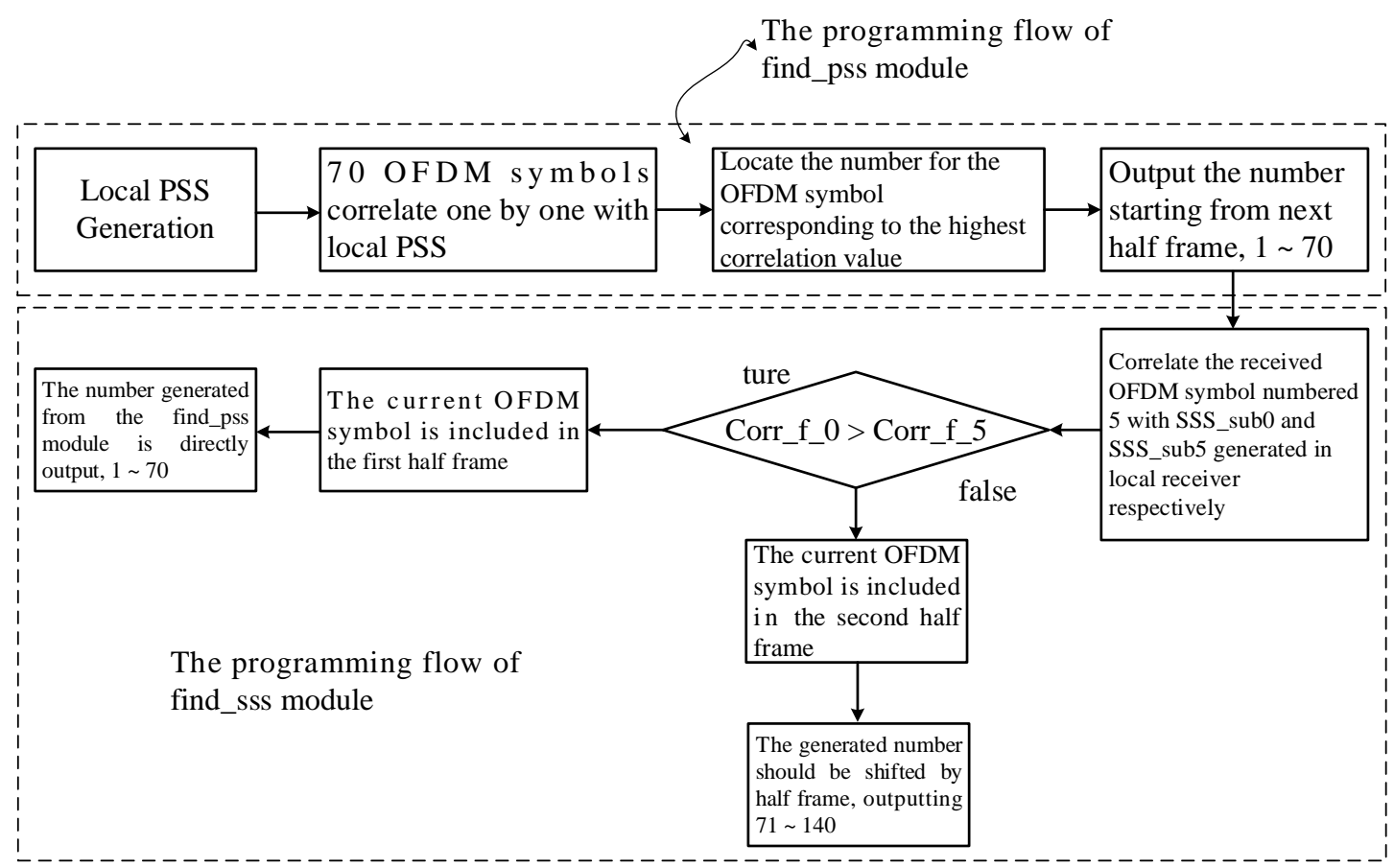

Figure 5. The Program Flow Chart of Synchronization

\subsection{The Calculation of the Physical Layer Cell ID}

The sequence of PSS/SSS is relative to physical layer cell ID and is used to identify cells by radio terminal. As demonstrated in Figure 6, we can see the calculation of the physical layer cell ID. There are three kinds of PSS sequences, referring to three possible values of $N_{I D}^{(2)}, N_{I D}^{(2)}$, namely $, 0,1$ and 2. There are 168 kinds of SSS sequences, referring to the values of $N_{I D}^{(1)}, N_{I D}^{(1)}$ varying from 0 to 167 . In the frame structure of FDD Type 1, PSS/SSS locates in both subframe 0 and subframe 5, and in two consecutive OFDM symbols.

While using the received the data of the half frame, 70 OFDM symbols, the receiver correlates them one by one with the three kinds of PSS sequences generated in local receiver, resulting in 210 correlation values, and then compares them. If the highest correlation value is among the first 70 values, the $N_{I D}^{(2)}$ is 0 . If the highest value is among the middle 70 ones, the $N_{I D}^{(2)}$ is 1 . If the highest value is among the last 70 ones, the $N_{I D}^{(2)}$ is 2. Completing the above steps, we can have the value of $N_{I D}^{(2)}$.

After the procession of the find_pss module, the half frame synchronization is achieved, so the receiver can number the received OFDM symbols from 1 to 70. Meanwhile, it locates the PSS included in the current half frame. From the Figure 4, the OFDM symbol including PSS is numbered 6, and PSS/SSS is located in two consecutive OFDM symbols, thus, the number for SSS is 5 . The receiver correlates the OFDM symbol numbered 5 with 168 kinds of SSS sequences generated in local receiver one by one, obtaining 168 correlation values. By comparing them, we can get the highest correlation value. Correspondingly, in this situation, the SSS of OFDM symbol numbered 5 is the same with the kind of the 168 SSS sequences, which exactly corresponds to the highest correlation value. Then we can obtain the value of $N_{I D}^{(1)}$ ranging from 0 to 167.

After getting the values of $N_{I D}^{(1)}$ and $N_{I D}^{(2)}$, we can use Equation (2) to calculate 
physical layer cell ID- $N_{\text {cell }}^{I D}$, thus accomplish identifying cell by radio terminal through using PSS/SSS.

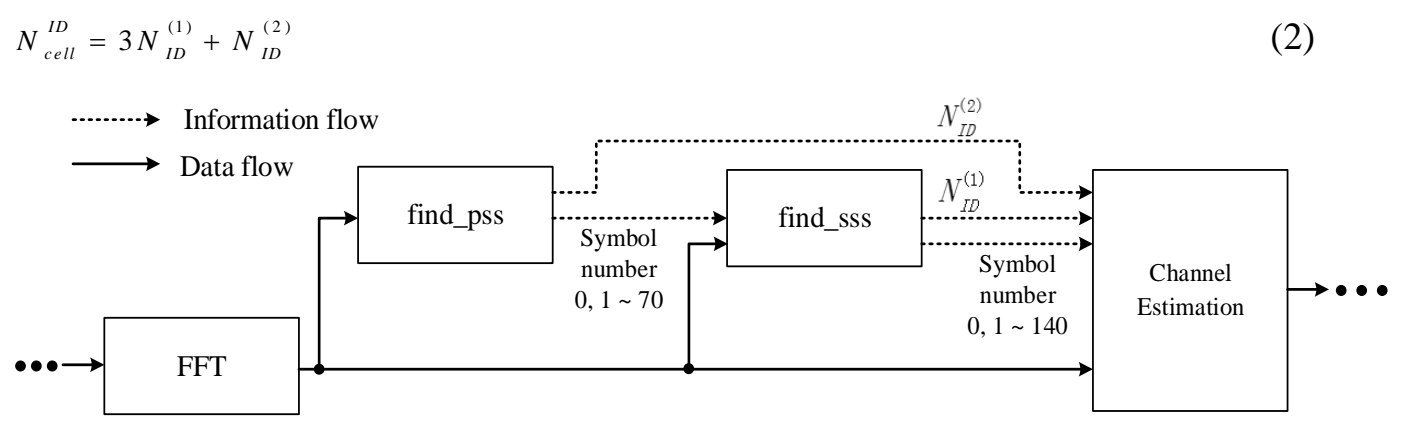

Figure 6. The Procedure of Calculating Physical Cell ID

\section{Test Results}

The test platform chooses USRP N210 from Ettus Research ${ }^{\mathrm{TM}}$ Company as the RF front-end for the BS and radio terminal. Using Dell Power Edge T620 as the Central Processing Unit (CPU), GNU Radio is installed into the CPU to process baseband signal. The transmit power of RF front-end is $46 \mathrm{dBm}$, system bandwidth is $10 \mathrm{MHz}$, central frequency is $2.4 \mathrm{GHz}$, the sampling rate of USRP $16.67 \mathrm{MHz}$ and antenna gain $3 \mathrm{dBi}$ with single antenna transmitting and receiving.

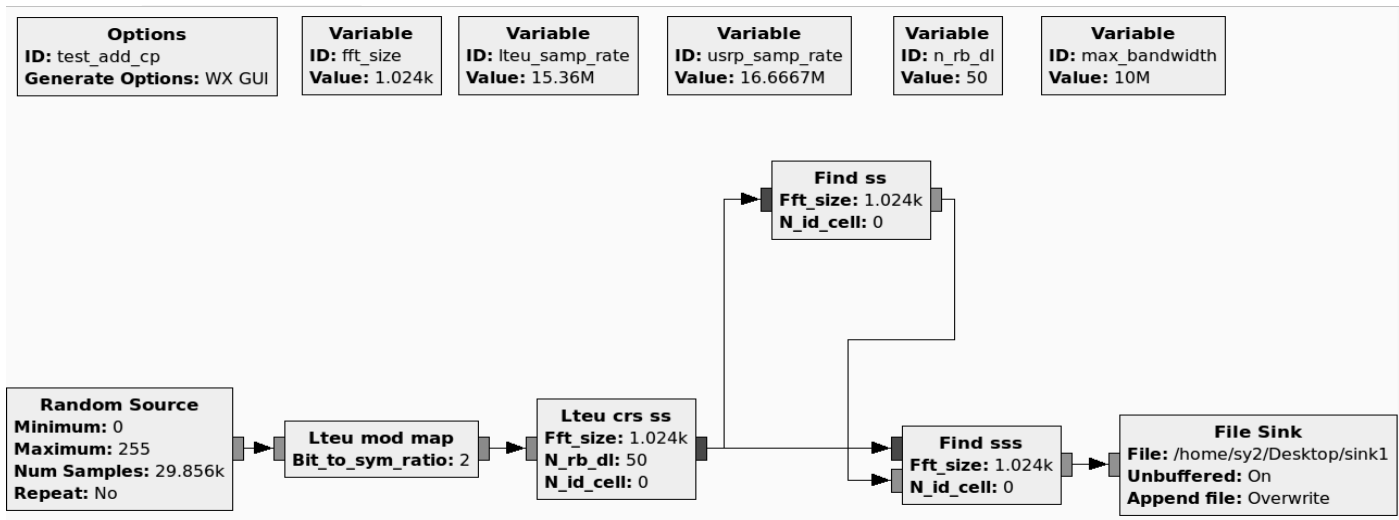

Figure 7. The Signal Processing Flow Chart of Test Platform

As shown in Figure 7, the find_sss module output OFDM symbol numbers one by one to the following modules. The find_pss and find_sss module consumes 70 OFDM symbols respectively, then the half frame and symbol synchronization can be achieved. Therefore, the 140 OFDM symbols included in the first radio frame should be output 140 zeros correspondingly.

In Figure 8, we can see the test results of Synchronization of the test platform. As shown in Fig. 8 (a), after the half frame and symbol synchronization, the receiver of the platform numbers the OFDM symbols correctly. In the process of the half frame and symbol synchronization, the receiver output 0 . After the synchronizations, the synchronous module output the numbers of OFDM symbols from 1 to 140 periodically. As demonstrated in Figure 8 (b), after the receiver finish numbering each OFDM symbol of one frame, it numbers that of the next frame from 1 to 140. Finally, the synchronization of transmitter and receiver of the platform is achieved. 

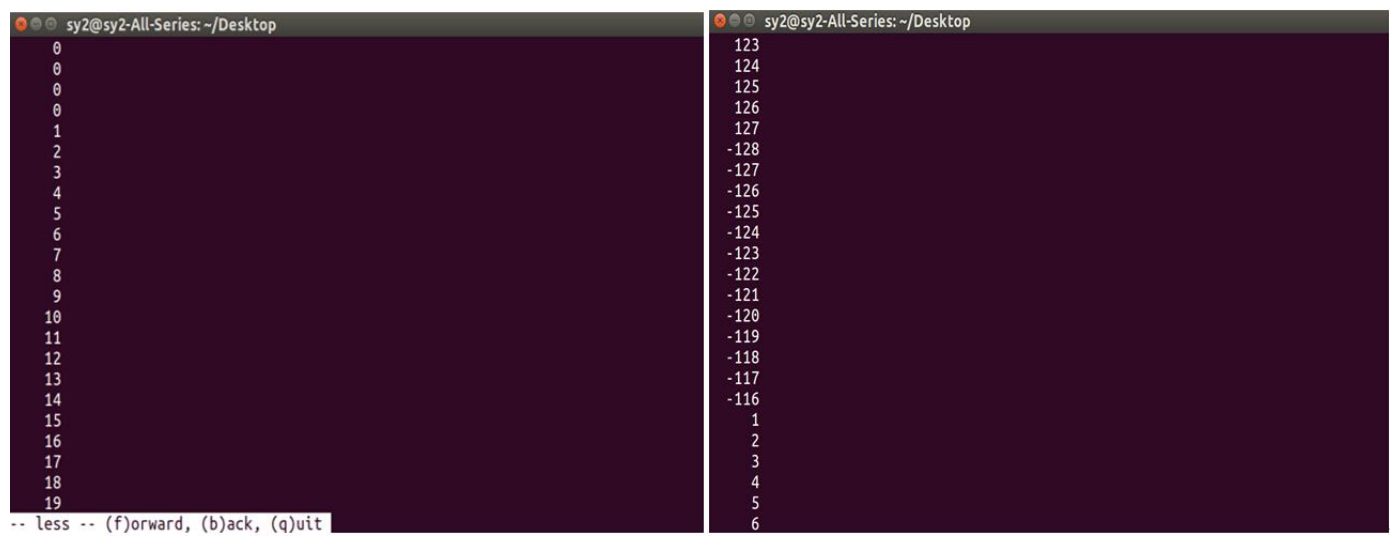

(a) Testing result of the synchronization of the first frame (b) Testing result of the synchronization during both the previous frame and the next one

Figure 8. The Test Results of Synchronization

We also develop a GUI to monitor the real-time system performance in the experiments. The GUI of platform is programmed by WxPython as shown in Figure 9. This figure is a snapshot of the real-time experiment results from GUI display when the platform is working. The received baseband signals, the demodulated signals before turbo decoding, the throughput, and the Bit Error Rate (BER) at each UE are shown from left to right, respectively. We use color blue to represent UE 1 and color red to represent UE 2. In addition, the green column in the throughput figure is the sum of the throughputs of UE 1 and UE 2 in order to represent the total system throughput. The experiment results demonstrate that both small cell UE 1 and macro cell UE 2 can receive the signals normally.

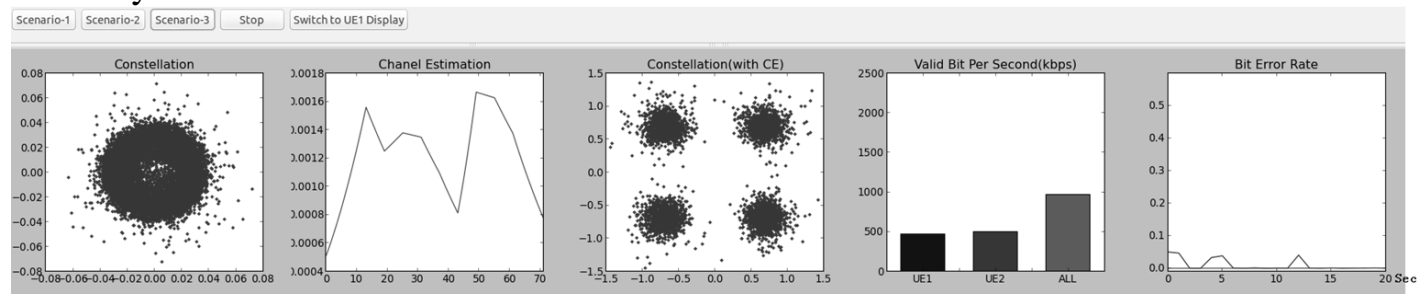

Figure 9. A Snapshot of the Real-Time Experiment Results from GUI

The throughput and BER of the users are two important performance indexes. Figure 10 shows the BER of users in Heterogeneous Networks (HetNet) without and with enhanced Inter-Cell Interference Coordination (eICIC). In this figure, the horizontal axis is about the change of time and the vertical axis shows that of BERs. Figure 11 shows the throughput of users in HetNet without and with eICIC. The horizontal axis reveals the change of time and the vertical axis shows that of user throughputs.

In the situation without eICIC, the communication rates of both small cell UE 1 and macro cell UE 2 are about $450 \mathrm{Kbps}$. However, the BER of UEs is as high as 30-40\% due to the inter-cell interference. Therefore, UEs cannot communicate properly. When the eICIC is used at about the $10^{\text {th }}$ second, user BER drops significantly. At the same time, the throughput of users decreases slightly from that without eICIC to about $300 \mathrm{kbps}$ because there are some subframes carrying no data. The slight decline of throughput of the users causes the obvious decrease of BER. The throughput of the users decline slightly and the BER decreases obviously, in return. 


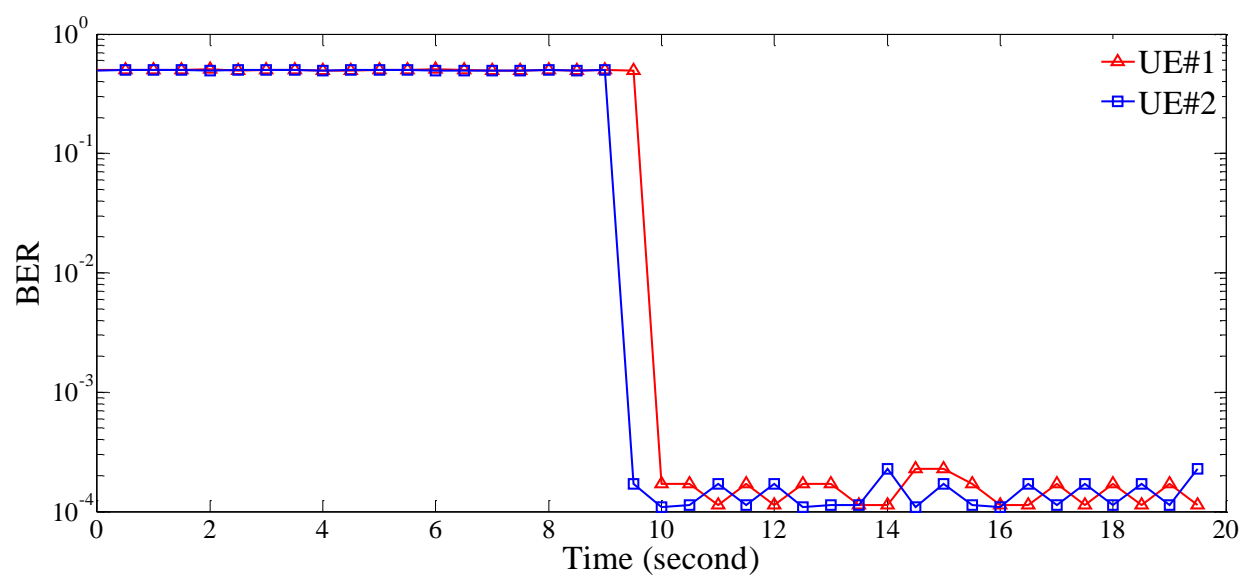

Figure 10. The Contrast of BERs Without and With eICIC

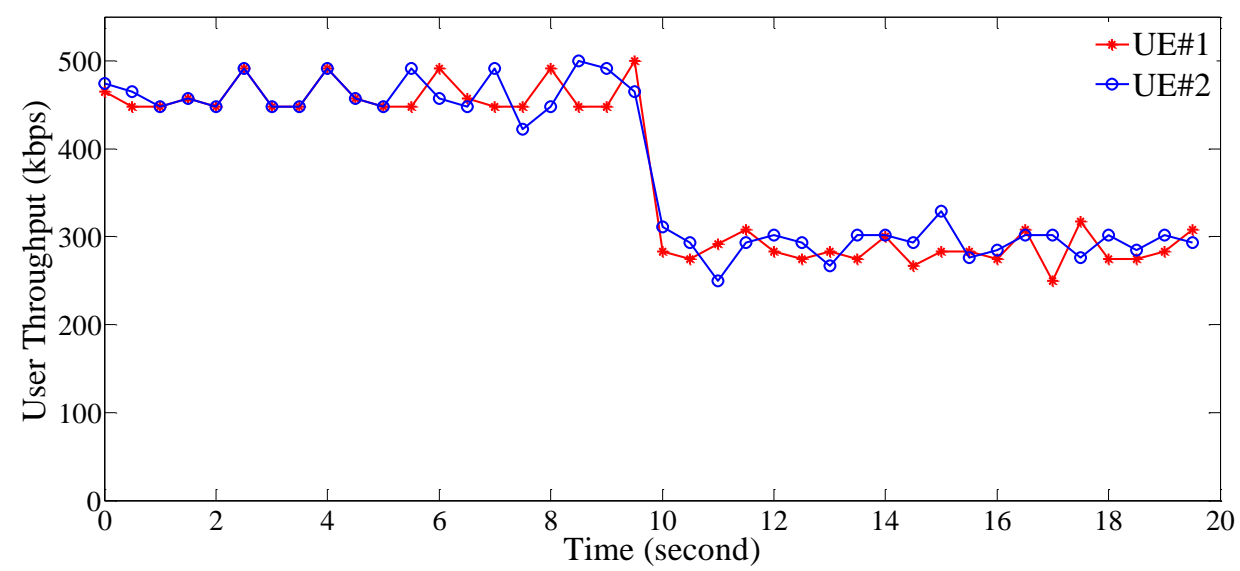

Figure 11. The Contrast of User Throughputs Without and With eICIC

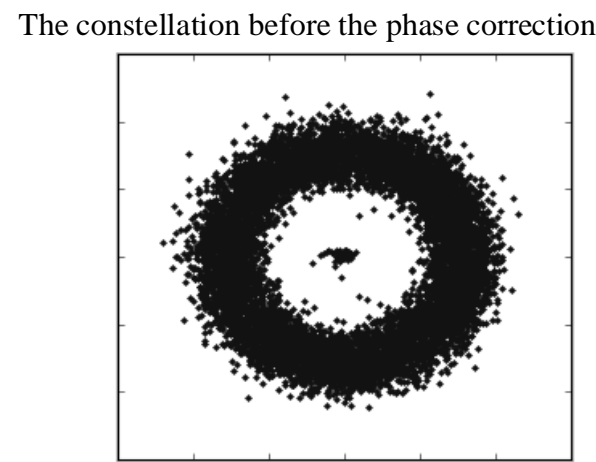

(a)
The constellation after the phase correction

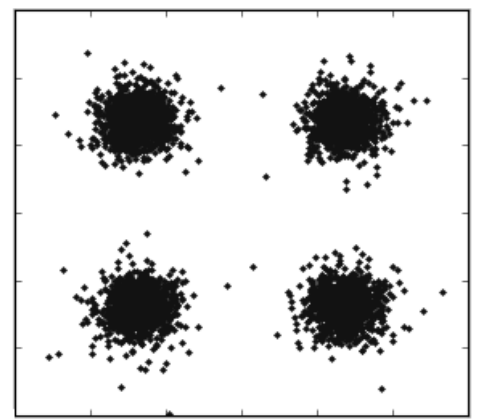

(b)

\section{Figure 12. The Constellations Before and After the Phase Correction}

Figure 12 shows the constellations before and after the phase correction. Platform uses QPSK modulation. After synchronization and Fast Fourier Transformation (FFT), the receiver obtains a frame data. The positioning error of the FFT window leads to the rotation of constellation point. While the Inter-Carrier Interference (ICI) leads to cloud divergence of constellation. As a result, the band-shaped ring is formed as in Figure 12 (a). After the phase correction is adopted, constellation points of the received data are 
gathered again in positions of $\pi / 4,3 \pi / 4,5 \pi / 4$ and $7 \pi / 4$ of the signal space.

\section{Conclusions}

We design and implement a test platform for real-time mobile communication technology. The platform is constructed by GNU Radio and USRP based on SDR. The platform has advantages of flexibility, general structure, modularity, extensibility, portability and being improved or extended easily, meaning that it can be used to test and verify the evolving mobile communication technologies. The operating result shows that the test platform achieves the synchronization of transmitter and receiver, and it also reflects real influence on the synchronization of the platform, which is exerted by the channel environment and interference.

\section{Acknowledgements}

We would like to thank the anonymous referees whose comments helped us to improve the presentation of this paper. This paper is funded by: the National Science Foundation of China (61302054); the Foundation of Chongqing University Postgraduates' Science and Innovation (CDJXS12161103); Scientific and Technological Research Program of Chongqing Municipal Education Commission (KJ1501107)

\section{References}

[1] White paper, "Cisco Visual Networking Index: Global Mobile Data Traffic Forecast Update", CISCO, (2014), pp. 2013-2018.

[2] E. Dahlman, S. Parkvall and J. Sköld, “4G-LTE/LTE-Advanced for mobile broadband. Academic Press, New York, (2011).

[3] A. Ghosh, R. Ratasuk, B. Mondal, N. Mangalvedhe and T. Thomas, "LTE-Advanced: next-generation wireless broadband technology.", IEEE Wireless Communications, vol. 3,no. 17, (2010).

[4] H. V. Balan, M. Segura, S. Deora, A. Michaloliakos, R. Rogalin, K. Psounis and G. Caire, "USC SDR, an easy-to-program, high data rate77", real time software radio platform. Proceedings of the Second Workshop on Software Radio Implementation Forum. (2013) August 25-30; Hong Kong, China

[5] TS 36.211. Evolved Universal Terrestrial Radio Access (E-UTRA); Physical Channels and Modulation. 3GPP, (2011)

[6] H. Wang, W. Jouini, A. Nafkha, J. Palicot, L. S. Cardoso and M. Debbah. Blind standard identification with bandwidth shape and GI recognition using USRP platform and SDR4all tools. Proceedings of the Fifth International Conference on Cognitive Radio Oriented Wireless Networks \& Communications. Cannes, French, (2010) June 1-5;

[7] Z. Huang, W. Wang and Y. Zhang, "Design and implementation of cognitive radio hardware platform based on USRP”, Proceedings of the 2011 IET International Conference on Communication Technology and Application; Beijing, China, (2011) October 160-164.

[8] White paper, C-RAN The Road Towards Green RAN. China Mobile Research Institute, (2011)

[9] GNU Radio Website, http://www.gnuradio.org.

[10] Ettus Research Website, http://www.ettus.com.

[11] Z. Zhao, G. Yang, Q. Liu, K. Li and L. Cui. Implementation and application of a multi-radio wireless sensor networks testbed. IET Wireless Sensor Systems, vol. 4, no.1, (2011).

[12] A. Ghosh, N. Mangalvedhe, R. Ratasuk, B. Mondal, M. Cudak, E. Visotsky, T. A. Thomas, J. G. Andrews, P. Xia, H. S. Jo, H. S. Dhillon and T. D. Novlan, "Heterogeneous cellular networks: from theory to practice", IEEE Communications Magazine, vol. 6, no. 50, (2012)

[13] Technical Document R1-130754. System simulation results on heterogeneous networks with cell range extension. Renesas Mobile Europe Ltd, (2013)

[14] TS 36.300. "Evolved universal terrestrial radio access (E-UTRA) and evolved universal terrestrial radio access network (E-UTRAN)”, overall description; stage, GPP, vol. 2, no. 3, (2012). 


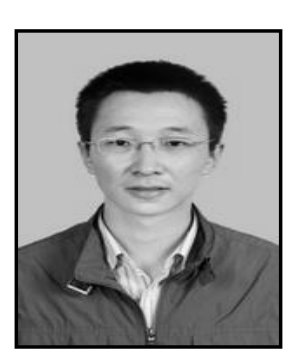

\section{Authors}

Peng Li received his Bachelor's degree in Electronic Science and Technology from Si Chuan University, Cheng Du, in 2004. He received his Master's degree in Communication and Information System from Chong Qing University, Chong Qing, in 2010. He has been studying Communication and Information System in College of Communication Engineering, Chongqing University, Chongqing, since 2012. He has actively contributed to design and implementation of LTE, LTE-Advanced physical layer and MAC layer by software defined radio. He is researching SDR, Cognitive Radio and LTE-Advanced HetNet. Since 2010, he has been working as an lecturer in School of Electronic and Electrical Engineering, Chongqing University of Arts and Sciences, Chongqing, China.

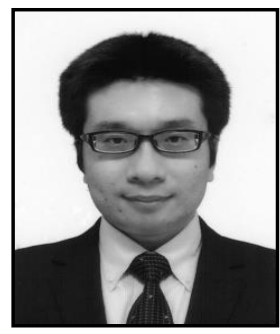

Yun-jian Jia received his B.S. degree from Nankai University, China, and his M.E. and Ph.D. degrees in Engineering from Osaka University, Japan, in 1999, 2003 and 2006, respectively. From 2006 to 2012, he was a Researcher with Central Research Laboratory, Hitachi, Ltd., where he engaged in research and development on wireless networks and contributed to LTE/LTE-Advanced standardization in 3GPP. He is now a professor at the School of Communication Engineering, Chongqing University, Chongqing, China. He is the author of more than 40 papers, more than 60 inventions, and more than 30 technical documents of $3 \mathrm{GPP}$. He is a member of IEEE and IEICE, and holds 19 patents. His research interests include multiple-antenna technologies, 4G and beyond wireless communications systems, resource management of mobile networks.

Fei Chen received his Bachelor's degree in Electronic Information Science and Technology from Central South University, China, in 2013. He is currently working toward the Master's degree in Communication and Information System in College of Communication Engineering, Chongqing University, Chongqing, China. He mainly engages in research of Software Defined Radio and Cognitive Radio.

Pei-hua He received her Bachelor's degree in College of Communication and Engineering in Chongqing University, China, in 2011. She is currently working toward the Doctor's degree in Circuits and System in the same college and university. She mainly engages in research and implementation of SDR real-time communication system, just like CW, PSK31 and parts of the physical links of LTE-Advanced. She is researching in algorithm of decoding the telemetry form the amateur radio satellite. She is also research the Frequency Division Multiplex Digital Voice (FDMDV) system implementation.

Hui-fang Fan received her Bachelor's degree in electronics engineering from the Communication Institute, China, her Master's degree in Signal and Information Processing from Chongqing University of Posts and Telecommunications, China, and the Doctor's degree in Circuits and System from Chongqing University, China, in 2000, 2009 and 2012 respectively. She is currently an associate professor of Chongqing Communication Institute, China. Her research efforts have been associated with statistical signal processing and detection of physiological recordings. She is working on the extraction of fetal cardiac signals. She has also worked in industry on the design and implementation of digital electronics and software in wireless body sensor networks. 